\title{
La puntura a occhiello della fistola artero-venosa: pro e contro
}

\author{
Sandro Galli, Alfio Zollo, Fosco Cavatorta* \\ U.O.C. Nefrologia e Dialisi, Ospedale di Imperia, Asl 1 imperiese, Imperia \\ *Già Direttore della Nefrologia e Dialisi di Imperia
}

\begin{abstract}
The BUtTONHOLE TEChNiQUe FOR FiSTUla CANNUlation: PROS AND CONS
Abstract. The most common method of arteriovenous fistula (AVF) cannulation is the standard rope-ladder technique. An alternative needling possibility is the buttonhole technique (BHT), by which needles are inserted exactly at the same position. Although earlier studies associated the BHT with a reduction in pain and hematoma formation, more recent randomized studies show no reduction in pain and an increased risk of local and systemic infection. However 1 trial with a follow-up of up to 1-year showed a better survival of the AVFs with the BHT, and did not confirm the increased risk of infection in the BHT group. The abovementioned study is the first well-constructed randomized clinical trial that directly compares the BHT with the use of BioHole peg, helping the development of the fibrous tract, and the rope-ladder cannulation techniques.

In any case, the successful implementation of the BHT requires the dialysis nurse to develop a high level of knowledge and several specific skills; it also requires frequent monitoring and a continuous evaluation and education regarding the use of the technique. We here emphasize the need for retraining the staff at specified time intervals to ensure that the proper technical skills are maintained. Therefore, in order to prevent BHT complications, a strict cannulation protocol should be followed.

Given the potential increased risk of infections associated with BHT, a better understanding of its actual benefit is needed. Clearly, further rigorous studies are needed, and these should focus on the role of the polycarbonate peg and of topical antimicrobial prophylaxis.
\end{abstract}

Key words: Buttonhole, Vascular access, Cannulation of arteriovenous fistula, Hemodialysis

Conflict of interest: None.

Financial support: None.

Accettato: 27 Gennaio 2014

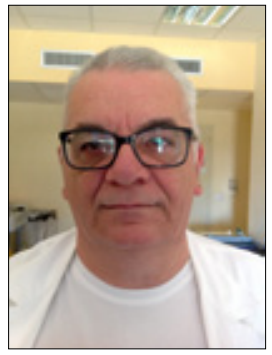

Sandro Galli

\section{Introduzione}

Di norma, la puntura della fistola arterovenosa (FAV) è sempre stata considerata un gesto "banale" da affidare spesso al personale infermieristico o a giovani medici e, per il perdurare di concezioni dal vago sapore medioevale, una pratica poco "nobile", come molte altre operazioni di tipo chirurgico, ovvero di tipo "manuense".

Questo atteggiamento, insieme a un rifiuto più o meno inconscio verso il nuovo e/o il cambiamento, spiega lo scarso interesse verso la venipuntura della fistola (1) $\mathrm{e}$, in particolare, verso possibili novità in questo campo. Solo negli ultimi anni la diffusione in molti paesi della diversa tecnica di puntura, la buttonhole technique (BHT), chiamata puntura a occhiello o ad asola o a sito costante, ha richiamato l'attenzione del mondo scientifico, con la comparsa di numerosi lavori dedicati a questa modalità di puntura della fistola $\mathrm{A}-\mathrm{V}$.
La segnalazione, tuttavia, di complicanze soprattutto di tipo settico in recenti lavori ha spinto l'amico e collega Dr. M. Napoli a fare una revisione della letteratura tra il 2011 e il 2013 su PubMed, traendo da questa analisi e in base anche alla sua grande esperienza nel campo degli accessi vascolari motivati dubbi sui possibili benefici della metodica e sui possibili rischi infettivi per la trasformazione della FAV in una sorta di catetere senza la presenza di un corpo estraneo.

Questo recentissimo articolo, comparso sul Giornale di Tecniche Nefrologiche e Dialitiche (1), oltre a essere ben documentato e di grande interesse per la pratica dialitica, offre l'occasione per rivedere la problematica legata alla BHT.

\section{Discussione}

Nell'articolo si sottolinea che la lunga esperienza acquisita da anni sui tunnel dei cateteri per la dialisi peritoneale (CP) e dei cateteri venosi centrali (CVC) per l'emodialisi avrebbe dovuto far prevedere la maggiore incidenza con la BHT rispetto alla puntura a scala di corda di infezioni locali e sistemiche, 
come è emerso nella maggior parte degli studi sia randomizzati che osservazionali, attribuita alla difficoltà di disinfettare adeguatamente il tunnel sottocutaneo.

Pur non potendo non condividere quanto sopra, è opportuno fare alcune considerazioni in merito.

Come spiegare il fatto che, in molti Centri, da anni e, in alcuni casi, sin dal 1970 (2), viene utilizzata la BHT senza gli inconvenienti segnalati, tanto da essere considerata la tecnica di prima scelta non solo nell'emodialisi domiciliare ma anche nell'emodialisi ospedaliera (3) e da essere consigliata dalle Linee Guida DOKI?

E, ancora, come spiegare i risultati contraddittori emersi da questi studi, ai quali potremmo aggiungere un piccolo trial randomizzato del 2010 condotto su 47 pazienti che riporta un basso livello di complicanze e una riduzione del dolore nei pazienti sottoposti a BHT (4)? Infatti, vi sono studi che documentano un aumento delle infezioni nei gruppi che praticano la BHT, senza riduzione del dolore da puntura e senza un aumento della sopravvivenza dell'accesso vascolare, e altri studi, anche se prevalentemente di tipo osservazionale, che, al contrario, segnalano una diminuzione degli ematomi e del dolore da puntura, senza un aumento nell'incidenza delle infezioni locali e sistemiche.

Di particolare interesse è il recente studio randomizzato con follow-up a un anno di Vaux et al. (5), condotto su 140 pazienti; si tratta, infatti, di uno studio eseguito, come sottolineato da Lindsay nel suo Editoriale (3), in modo corretto e dell'unico trial con l'uso del device, il policarbonato peg (PEG), che facilita la formazione del tunnel sottocutaneo. E, pertanto, possibile che gli ottimi risultati riportati, dall'assenza di infezioni nel gruppo BHT contro 2 casi nel gruppo con puntura a sito variabile alla maggiore sopravvivenza della FAV, possano dipendere dalla migliore formazione del tunnel con una più agevole puntura con l'ago non tagliente e la riduzione delle possibili complicanze legate alla manovra.

Bisogna, tuttavia, rimarcare che il periodo di osservazione di 12 mesi è probabilmente insufficiente per evidenziare il rischio infettivo.

Probabilmente, questi risultati contrastanti sono una conseguenza del diverso livello di "skill" degli operatori, per cui l'inadeguatezza nella preparazione del tunnel e nella manovra di incannulamento con l'ago smusso porterebbe ad aumentare invece che a ridurre le complicanze da puntura della FAV; la puntura con BHT non può essere affidata a un qualsiasi puntore, magari dopo un affrettato addestramento, che non sia sufficientemente abile nella puntura con l'ago smusso. La BHT, infatti, non è una semplice variante della puntura a sito variabile ma, al contrario, è una tecnica di incannulamento del vaso del tutto differente (5) e, pertanto, è da affidare solo a del personale idoneo e motivato, tenendo presente che la manualità con gli aghi smussi viene acquisita con la pratica e la costanza. Comunque, il fatto che si possa osservare un aumento delle infezioni nella BHT attesta come la metodica richieda un'attenzione verso le norme di asepsi di gran lunga superiore rispetto a quanto richiesto nella puntura a sito variabile. Come per i CVC e i cateteri peritoneali, il rigoroso rispetto dei tempi di disinfezione e l'accuratezza della disinfezione che va effettua sia prima che dopo la rimozione della crosta sono cru- ciali. Infatti, come giustamente sottolineato nell'articolo del Dr. Napoli, lo scorrimento dell'ago nel suo tragitto potrebbe trascinare nel torrente circolatorio detriti cellulari e batteri eventualmente non rimossi da una disinfezione non adeguata. A questo proposito, possiamo testimoniare di aver assistito all'inserimento degli aghi a punta smussa senza la seconda disinfezione dopo la rimozione della crosta e questo, probabilmente, per l'erronea convinzione dell'operatore di aver disinfettato il campo operatorio compresa la crosta!

A conferma di quanto detto sopra, vi è l'esperienza di Imperia, dove la BHT è stata iniziata nel Giugno del 2006 e progressivamente estesa negli anni seguenti alla maggior parte dei pazienti con buoni risultati, in particolare senza complicanze infettive e con un alto gradimento da parte del personale e dei pazienti. Tuttavia, negli ultimi 2 anni, 2012 e 2013, si sono verificati alcuni casi di gravi complicanze infettive da stafilococco aureo (endocardite e spondilodiscite), il tutto in concomitanza con un elevato turnover del personale infermieristico e una riorganizzazione dirigenziale e con la conseguente perdita della figura medica di riferimento per la BHT.

L'esperienza di Imperia sta a dimostrare che il mancato rispetto di determinate regole espone inevitabilmente a un aumentato rischio infettivo. Molteplici sono le cause: 1'estensione della tecnica a tutto il personale di assistenza, l'elevata rotazione del personale con lo spostamento di infermieri particolarmente addestrati e abili nel loro lavoro, l'aumento probabilmente eccessivo del numero dei pazienti trasferiti a questa tecnica, il ritmo di lavoro "stressante", specialmente nella fase di attacco e stacco, simile, nei grandi Centri Dialisi, alla catena di montaggio delle fabbriche, e la progressiva perdita di attenzione alle procedure legata alla ripetitività della manovra.

È opportuno, pertanto, ribadire un punto fondamentale, e, precisamente, che la BHT non è una semplice variante della puntura a scala di corda ma è una tecnica del tutto diversa, al punto da richiedere non solo una riorganizzazione del lavoro e dei compiti dello staff ma, soprattutto, un vero e proprio cambiamento culturale che dovrebbe riguardare non solo il personale addetto ma anche la Dirigenza Medico-Infermieristica, la Direzione Sanitaria e l'organizzazione sindacale.

L'esperienza acquisita e l'attenta valutazione dei lavori pubblicati ci porta a proporre alcuni suggerimenti.

\section{I raccomandazione:}

In considerazione del fatto che non tutti siamo idonei a praticare manovre chirurgiche non possedendone le necessarie qualità, così come non tutti siamo idonei, per esempio, a fare i piloti di aerei, la BHT andrebbe affidata a un numero limitato di infermieri e medici particolarmente motivati e addestrati, obiettivo più facilmente raggiungibile in Centri di modeste dimensioni, evitando di estendere la tecnica a tutti i pazienti. Di notevole utilità sarebbe l'istituzione della figura dell'infermiera degli accessi vascolari (Vascular Access Nurse, VAN), come nell'Unità Operativa di Emodialisi dell'Ospedale Malpighi di Bologna, o, come consigliato da Van Loon et al. (6), di quella di venipuntori ai quali affidare l'uso dell'ago tagliente, con il compito non solo di formare nelle prime settimane il tunnel, ma anche di procedere alla puntura 
in caso di insuccesso con l'ago smusso. Consigliabile adottare il metodo del Dr. Toma (7), con l'utilizzo del device, il policarbonato peg, nella fase di formazione del tunnel, che può richiedere da 2 a 4 settimane e l'uso, anche in questa fase, di aghi a punta smussa.

\section{II raccomandazione:}

Gli inconvenienti emersi in questi studi non sarebbero imputabili alla tecnica in sé ma, piuttosto, a un'inadeguata applicazione delle procedure da parte degli operatori $(8,9)$, per cui, considerando l'inevitabile perdita progressiva nel tempo della dovuta attenzione e della meticolosità richiesta, diventano indispensabili un continuo controllo del rispetto delle procedure e il periodico addestramento del personale addetto (1-2 volte all'anno). Ricordiamo alcuni passaggi fondamentali e assolutamente obbligatori che spesso, tuttavia, non vengono rispettati, come il lavaggio del braccio, l'ammorbidimento della crosta mediante garze intrise di disinfettante prima dell'inizio della seduta e/o mediante creme antisettiche o anestetiche posizionate dal paziente mezz'ora prima della dialisi, l'attenta e completa rimozione della crosta con un ago sterile e, soprattutto, la ri-disinfezione dei siti di puntura con un tempo di contatto più protratto del solito. In considerazione degli ottimi risultati ottenuti con l'uso del bactroban (10), potrebbe essere opportuno effettuare la profilassi con l'applicazione locale di mupirocina.

\section{III raccomandazione:}

La BHT è particolarmente indicata in pazienti selezionati con FAV di difficile puntura per la profondità o la tortuosità del vaso o con un tratto di vena troppo corto per la puntura a sito variabile, situazione che porta inevitabilmente a praticare la puntura cosiddetta ad area. In questi casi, è possibile praticare la BHT anche con un monoago a punta smussa, il che può consentire l'utilizzo di tratti anche molto brevi di una FAV funzionante, evitando revisioni chirurgiche con l'eventuale trasposizione e/o la superficializzazione del vaso venoso. Inoltre, la BHT aiuta a risolvere situazioni di ansia da prestazione nel personale e di ansia da risultato nel paziente, riducendo, nello stesso tempo, anche il dolore legato alla manovra e il possibile stravaso di sangue, con lo sviluppo di ematomi più o meno estesi. Infatti, nel caso dell'effetto rimbalzo o di difficoltà nell'attraversamento del tunnel con l'ago smusso, si dovrebbe evitare di forzare lacerando la parete del tragitto sottocutaneo e creando false vie, possibili ricettacoli di germi.

\section{Conclusioni}

L'aumento del rischio di infezioni locali e sistemiche segnalato in molti lavori recenti, se, da un lato, rende comprensibile la diffidenza da parte del medico verso questa "nuova" tecnica di venipuntura della FAV nel timore di aumentare il rischio infettivo nel suo Centro, dall'altro non deve portare a buttare via l'acqua sporca con il bambino.

Infatti è indubbio, come attestato da vari lavori non solo di tipo osservazionale, che la BHT, se praticata in modo corret- to e con un periodico addestramento del personale selezionato, garantisce in molti pazienti, specialmente in quelli con un accesso vascolare difficoltoso, una migliore qualità della vita e, probabilmente, anche una maggiore sopravvivenza dell'accesso. Bisogna, però, sempre tenere presente che, dal momento che la BHT costituisce una possibile fonte di infezione per ovvi motivi, non si dovrà mai abbassare la guardia, evitando di cadere nella pericolosa routine, pena un aumento delle complicanze settiche nel Centro. Non vi è alcun dubbio che sono necessari ulteriori studi randomizzati e controllati; tuttavia, solo studi condotti con modalità ben precise e specifiche potranno fare luce su questo problema. Gli Autori, infatti, ritengono opportuno uno studio multicentrico randomizzato condotto con l'utilizzo del PEG nella fase di formazione del tunnel e di un numero limitato di pungitori in grado di praticare la puntura non solo con l'ago tagliente ma anche con l'ago smusso.

\section{Riassunto}

La tecnica buttonhole (BHT), proposta sin dagli anni ' 70 in alternativa alla più comune modalità di puntura detta a sito variabile, in questi ultimi anni è stata adottata in molti Centri Dialisi in Europa e anche in Italia come preferibile modalità di incannulamento perché associata a minori complicanze. Tuttavia, la segnalazione in recenti studi di tipo osservazionale ma, soprattutto, in 2 su 3 studi randomizzati di un aumento dell'incidenza di infezioni locali e sistemiche nei pazienti sottoposti a BHT ha sollevato dubbi sulla sicurezza della tecnica. Vengono analizzate le possibili cause alla base dei risultati contraddittori che emergono da questi studi, rilevando come il trial randomizzato, dove non viene riscontrato con un follow-up di 1 anno un aumentato rischio infettivo, sia stato l'unico a utilizzare il metodo del Dr. Toma, che prevede, per la creazione del tunnel per la puntura, l'inserimento di un particolare "device" in policarbonato (PEG) e l'uso, dopo la prima puntura, dell'ago a punta smussa. Dopo aver dato alcuni suggerimenti utili a ridurre al minimo le complicanze infettive sia locali che sistemiche, richiamando soprattutto all'assoluto rispetto delle procedure, si conclude che la BHT, purché praticata in modo corretto, possa essere utilizzata al pari dell'altra tecnica e sia preferibile in determinati casi.

$\mathrm{Si}$ auspicano ulteriori studi randomizzati da condurre, tuttavia, con l'uso del PEG, con un'adeguata selezione degli operatori e per periodi di osservazione più lunghi.

Parole chiave: Puntura a occhiello, Accesso vascolare, Puntura della fistola artero-venosa, Emodialisi

Dichiarazione di conflitto di interessi: Gli Autori dichiarano di non avere conflitto di interesse.

Contributi economici agli Autori: Gli Autori dichiarano di non aver ricevuto sponsorizzazioni economiche per la preparazione dell'articolo. 
Indirizzo degli Autori:

Dr. Fosco Cavatorta

U.O.C. Nefrologia e Dialisi

Ospedale di Imperia

Asl 1 imperiense

Via S. Agata 57

18100 Imperia

fosco.cavatorta@libero.it

\section{Bibliografia}

1. Napoli M. Il buttonhole: come trasformare una fistola in un catetere. Giornale di Tecniche Nefrologiche \& Dialitiche 2013; 25: 290-2.

2. Twardowski ZJ, Kubara H. Different sites versus constant sites of needle insertion into arteriovenous fistulas for treatment by repeated dialysis. Dial Transplant 1979; 8: 978-80.

3. Lindsay R. Should the Buttonhole Cannulation Technique Be Regarded as the Cannulation Method of Choice for Arteriovenous Fistulas? Am J Kidney Dis 2013; 62 (1): 7-9.
4. Struthers J, Allan A, Peer RK, Lambie SH. Buttonhole needling of arteriovenous fistulae: a randomized controlled trial. Asaio J 2010; 56 (4): 319-22.

5. Vaux E, King J, Lloyd S, et al. Effect of buttonhole cannulation with a polycarbonate PEG on in-center hemodialysis fistula outcomes: a randomized controlled trial. Am J Kidney Dis 2013; 62 (1): 81-8.

6. Van Loon MM, Goovaerts T, Kessels AG, et al. Buttonhole needling of haemodialysis arteriovenous fistulae results in less complications and interventions compared to the rope-ladder technique. Nephrol Dial Transplant 2010; 25: 225-30.

7. Toma S, Shinzato T, Fukui H, et al. A timesaving method to create a fixed puncture route for the buttonhole technique. Nephrol Dial Transplant 2003; 18: 2118-21.

8. Ball LK. The buttonhole technique for arteriovenous fistulas cannulation. Nephrol Nurs J 2006; 33: 299-304.

9. Pegoraro M. La puntura a occhiello della fistola arterovenosa. Storia e dati di evidenza nell'applicazione della tecnica. Giornale di Tecniche Nefrologiche \& Dialitiche 2008; 20: 1-6.

10. Nesrallah GE, Cuerden M, Wong J, Pierratos A. Staphulococcus aureus bacteremia and buttonhole cannulation: long-term safety and efficacy of mupirocin prophylaxis. Clin J Am Soc Nephrol 2010; 5: 1047-53. 